\title{
Novel anti-glioblastoma agents and therapeutic combinations identified from a collection of FDA approved drugs
}

Pengfei Jiang ${ }^{1}$, Rajesh Mukthavavam ${ }^{1}$, Ying Chao ${ }^{1}$, Ila Sri Bharati ${ }^{1}$, Valentina Fogal ${ }^{1}$, Sandra Pastorino ${ }^{1}$, Xiuli Cong ${ }^{3}$, Natsuko Nomura', Matt Gallagher ${ }^{1}$, Taher Abbasi ${ }^{4}$, Shireen Vali ${ }^{4}$, Sandeep C Pingle ${ }^{1}$, Milan Makale ${ }^{1}$ and Santosh Kesari ${ }^{1,2,3^{*}}$

\begin{abstract}
Background: Glioblastoma (GBM) is a therapeutic challenge, associated with high mortality. More effective GBM therapeutic options are urgently needed. Hence, we screened a large multi-class drug panel comprising the $\mathrm{NIH}$ clinical collection (NCC) that includes 446 FDA-approved drugs, with the goal of identifying new GBM therapeutics for rapid entry into clinical trials for GBM.

Methods: Screens using human GBM cell lines revealed 22 drugs with potent anti-GBM activity, including serotonergic blockers, cholesterol-lowering agents (statins), antineoplastics, anti-infective, anti-inflammatories, and hormonal modulators. We tested the 8 most potent drugs using patient-derived GBM cancer stem cell-like lines. Notably, the statins were active in vitro; they inhibited GBM cell proliferation and induced cellular autophagy. Moreover, the statins enhanced, by 40-70 fold, the pro-apoptotic activity of irinotecan, a topoisomerase 1 inhibitor currently used to treat a variety of cancers including GBM. Our data suggest that the mechanism of action of statins was prevention of multi-drug resistance protein MDR-1 glycosylation. This drug combination was synergistic in inhibiting tumor growth in vivo. Compared to animals treated with high dose irinotecan, the drug combination showed significantly less toxicity.
\end{abstract}

Results: Our data identifies a novel combination from among FDA-approved drugs. In addition, this combination is safer and well tolerated compared to single agent irinotecan.

Conclusions: Our study newly identifies several FDA-approved compounds that may potentially be useful in GBM treatment. Our findings provide the basis for the rational combination of statins and topoisomerase inhibitors in GBM.

Keywords: Glioblastoma, Drug screening, Patient-derived glioblastoma cell lines, Rational combination

\section{Introduction}

Novel therapeutic options are sorely needed to target glioblastoma (GBM), a notoriously treatment-resistant brain cancer. GBM is a leading cause of cancer-related death in the pediatric and adult populations, with most patients succumbing within 1-2 years [1,2]. The standard therapies are inadequate, and their toxicities lead to severe life-long morbidity in the small number of patients that survive [2]. Despite this grim prognosis, GBM is an

\footnotetext{
* Correspondence: skesari@ucsd.edu

${ }^{1}$ Translational Neuro-Oncology Laboratories, Moores Cancer Center, UC San Diego, La Jolla, CA 92093, USA

2Department of Neurosciences, UC San Diego, La Jolla, CA 92093, USA

Full list of author information is available at the end of the article
}

orphan disease that is in general not a priority for new drug development [3-5]. Moreover, the biology of GBM is complex and much remains to be learned about the putative key signaling pathways before they can be therapeutically exploited [6]. In view of the unmet and urgent clinical need, we were motivated to pursue recent data indicating that GBM may respond to some FDA-approved agents not previously identified as GBM therapeutics $[7,8]$. The in vitro screening of a broad range of drugs already approved for other indications is attractive as in vivo toxicity and pharmacology are well defined, and such compounds can enter GBM clinical trials rapidly either as single agents or as combinations. 
Accordingly, our goals were to identify and characterize single and combination agents having anti-GBM activity that we can potentially introduce into clinical trials quickly. To this end, using GBM cell lines and patientderived GBM cell cultures, we screened a 446-compound NIH Clinical Collection (NCC) library comprising FDAapproved drugs. To further improve the anti-GBM potency of these drugs, we tested various drug combinations and compared them to single drug treatment.

Our screening strategy included multiple human GBM cell lines of different origins in order to provide cross-validation of findings. These cell lines included 4 established serum-grown, immortalized human GBM lines, 4 patient-derived stem cell like GBM primary cells grown as neurospheres, and 2 primary patient-derived GBM lines grown as adherent cultures [9-14].

We did not confine our screening to only adherent GBM stem cell lines despite reports claiming that such lines remain undifferentiated longer and constitute a simpler, less variable assay $[11,14]$. It is not yet firmly established that the major therapeutic target in GBM is just the cancer stem cell tumor compartment and there are indications that other cell types within GBM may assume stem cell characteristics through genetic or epigenetic events [14-16]. In contrast to a single type or lineage of cells, neurospheres contain a mix of GBM stem cells and differentiated cells, which is more reflective of the composition of human GBM tumors [17]. Further, when dissociated neurospheres are implanted orthotopically, they are highly tumorigenic and authentically recapitulate the invasive natural history, composition, and histology of GBMs growing in humans [16]. Hence we report the identification of NCC active compounds through our screening approach on patient-derived stem cell-like GBM primary cells.

Our initial screening identified 22 compounds active against GBM ( $>50 \%$ cell death) [18] at pharmacological doses. These 22 compounds encompassed 11 drug classes. In particular, we found that the statin, pitavastatin, effectively induced cellular autophagy and suppressed tumor cell MDR-1 protein, to impressively enhance the potency of irinotecan, a topoisomerase 1 inhibitor used in cancer treatment [19-21]. This work newly identifies FDA approved drugs and drug combinations, notably pitavastatin and irinotecan, that may be useful for GBM treatment, and draws attention to the potential value of in vitro screening of approved compounds not currently used to treat GBM.

\section{Materials and methods}

Overview of cell-based (in vitro) screening for potential anti-GBM compounds

We acquired 446 small molecules that completed human clinical trials from the NIH Clinical Collection (NCC).
The initial broad screen was performed on U87 cells plated at 2000 cell per well on 96-well plates incubated overnight. All compounds were added to the plates to attain a final concentration of $10 \mu \mathrm{M}$. After 72 hours of incubation with drugs, the inhibition of cell proliferation was quantified by the Alamar Blue viability assay. Briefly, after incubation, Alamar Blue (\#BUF012B, AbD Serotec) was added directly to the culture medium, and the fluorescence measured at 560/90 to determine the number of viable cells (Infinite M200, Tecan Group Ltd.). The $\mathrm{IC}_{50}$ values were calculated using commercially available software (Prism ${ }^{\oplus}$, Graphpad Software, La Jolla, CA). We defined active compounds as those eliciting a greater than $50 \%$ reduction of cell viability in three independent screens. The 15 most potent and available drugs or compounds were then re-screened with other established glioma cell lines (LN443, A172 and U118), with the four patient derived GBM stem cell like primary neurosphere lines (SK72, SK262, SK429 and SK660), and with 2 GBM stem cell like primary cells (SK72 and SK262) grown as adherent culture. Pitavastatin was also tested in combinations with the other 12 compounds. The $\mathrm{IC}_{50}$ values were determined with and without pitavastatin $(2 \mu \mathrm{M})$, using the Alamar blue assay as described above.

\section{Isolation, culture, and compound activity testing with patient derived GBM cells Human GBM samples}

Fresh human GBM material was acquired from 4 GBM surgical patients and cultured as previously reported $[9,13]$. Briefly, the dissociated tissue was washed, filtered through a $30 \mu \mathrm{m}$ mesh and plated onto ultra-low adherence flasks at a concentration of 500,000 to $1,500,000$ viable cells $/ \mathrm{ml}$. The stem cell isolation medium included human recombinant EGF (20 ng/ml), human bFGF (10 ng/ml) and heparin $(2 \mu \mathrm{g} / \mathrm{ml})$. Sphere cultures were then passaged by dissociation, washed, resuspended in neural stem cell culture medium (\#05750,Stemcell Technologies), and plated on ultra low-adherence 96 well plates at 2000 cells per well for all subsequent drug testing.

Alternatively, patient-derived dissociated GBM tissues were plated onto laminin-1 coated plates (Sigma, 3-5 $\mu \mathrm{g} / \mathrm{ml}$ ). Cell populations were dissociated using Acutase (Sigma) and expanded for 5-10 passages, then plated on flat bottom for drug testing.

\section{Confirmation of stem cell marker expression}

Primary neurospheres were cytospun onto glass slides. Adherent primary cultures were grown onto Permanox ${ }^{\mathrm{Tm}}$ chamber slides (\#70380,EMS). Cells were incubated with human Nestin antibody (M1259, R\&D system) and then with fluorescein-labeled secondary antibodies, then stained with DAPI. The cells were visualized under a UV microscope (Olympus BX51). 


\section{Drug testing and survival assay}

As explained above, cells were seeded onto either regular or ultra-low adherence 96 well plates and incubated for 18-24 hours and then treated with vehicle control or single drugs or drug combinations. After 96 hours of incubation, Alamar Blue was added directly to the culture medium, and the fluorescence measured at 560/90 after 4-12 hours to determine the number of viable cells. The $\mathrm{IC}_{50}$ was calculated.

\section{Prediction of blood brain barrier (BBB) permeation by active compounds}

Although ample evidence has demonstrated that drugs of virtually any size or chemotype can enter brain tumor via leaky tumor microvessels, the ability to penetrate the intact blood brain barrier (BBB) is reasonably hypothesized to be useful for treating tumor cells infiltrating normal brain tissue along fiber tracts [22-25]. Hence we estimated the capacity of active anti-GBM compounds to cross the $\mathrm{BBB}$. We used standard software to calculate the $\log \mathrm{BB}$ value: $\log \mathrm{BB}=-0.0148 \mathrm{PSA}+0.152 \mathrm{CLog} \mathrm{P}+$ 0.139 ; PSA = polar surface area, $\mathrm{p}=$ octanol $/$ water partition coefficient $[26,27]$.

\section{Determination of cell cycle, autophagy, and apoptosis Cell cycle analysis}

GBM cells were seeded into $10 \mathrm{~cm}$ dishes at a density of $1 \times 10^{6}$, cultured overnight followed by the addition of $3 \mu \mathrm{M}$ pitavastatin with 24 or 48 hours of incubation. Cells were trypsinized and fixed in $70 \%$ ethanol for 30 minutes, incubated with $25 \mu \mathrm{g} / \mathrm{ml}$ propidium iodide (PI) and $250 \mu \mathrm{g} / \mathrm{ml}$ RNAase in PBS for 1 hour at $50^{\circ} \mathrm{C}$. After PI staining, cells were analyzed via flow cytometry (Canto, BD FACS), and the percentage of cells in $G_{0} / G_{1}$, $\mathrm{S}$ and $\mathrm{G}_{2}$ phases were calculated by ModFit LT software version 3.0.

\section{Detection of caspase activity}

Caspase-3 activity was measured with the Invitrogen Enzcheck caspase- 3 assay kit \#2 according to the manufacture's protocol. Briefly, $3 \times 10^{6}$ U118 cell were cultured and pitavastatin, irinotecan or the combination was added to the medium for 12 or 24 hours. Then $10^{6}$ cells were lysed, DEVD-R110 fluorescence substrate was added, and the fluorescence signal was measured and compared with a standard curve (Infinite M200, Tecan). Caspase $3 / 7$ activity was measured by the Apo-One caspase3/7 Kit (\#G7790, Promega). 20,000 cells were seeded on to 24 well plates, pitavastatin and vehicle were added, followed by incubation $(8,12,24$ and 48 hours) and caspase 3/7 activity was measured using a fluorescence-based substrate.

\section{Detection of autophagy markers-GFPLC3 punctuation}

Retrovirus carrying the GFPLC3 was produced by transfecting the 293GP2 cells with the pVSV-G and pBABEpuro-GFPLC3 plasmids (GFP fused with LC3 gene at the N-terminal, Addgene \#22405). Retroviral supernatants were harvested 48 hours later. U87, U118, U251 cells were seeded at a density of $2 \times 10^{5}$ in 6 well plates and infected $24 \mathrm{hr}$ later with the VSV-G/GFPLC3 virus. Stable cell lines were selected for 1 week in $1 \mu \mathrm{g} / \mathrm{ml}$ puromycin. GFPLC3expressing lines were seeded onto 24 well plates and treated with $1 \mu \mathrm{M}$ pitavastatin for 48 hours. Presence of GFPLC3 punctuation, which is a marker of autophagy was detected by UV microscopy (Olympus IX81).

\section{Western blot analysis for autophagy, apoptosis, and multidrug resistance protein}

LC3 (autophagy), caspase-3 (apoptosis), and MDR-1 (multidrug resistance protein 1 or P-glycoprotein 1,ABCB1) and tubulin (as loading control) were detected by western blotting following drug treatment. Cell lysates (10-50 $\mu \mathrm{g}$ protein) were loaded on to either 14\% SDS-PAGE gel (for LC3) or $4-12 \%$ gel (for caspase-3, and MDR-1), proteins transferred to PVDF membrane and probed with primary antibodies (L8918 Sigma, 9662 Cell signaling, Sc-8313 Santa Cruz, T4026 Sigma). The resultant protein bands were visualized by a supersignal kit (\#1856136,Thermo Scientific) after incubation with HRP-labeled secondary antibodies.

\section{Multi-drug resistance assay}

A cell-based fluorescence assay kit (Cayman Chemical Company, MI, USA, \#600370) was used to evaluate modulation of the MDR-1 protein by drugs. Calcein AM is a hydrophobic non-fluorescent dye that easily permeates living cells. The hydrolysis of Calcein AM by intracellular esterases produces calcein, a hydrophilic strongly fluorescent compound which is retained in the cell cytoplasm and can be measured using excitation and emission wavelengths at $485 \mathrm{~nm}$ and $535 \mathrm{~nm}$, respectively. Calcein AM is a substrate of MDR-1 protein P-gp, which causes its rapid extrusion from the plasma membrane, preventing accumulation of the fluorescent calcein inside the cytoplasm. Therefore measurement of fluorescent calcein allows for detection of MDR activity in live cells. Hoechst Dye staining of nuclei measured using of excitation and emission wavelengths $355 \mathrm{~nm}$ and $465 \mathrm{~nm}$ respectively to normalize cell numbers in well. GBM cells were seeded at $5 \times 10^{4} /$ well overnight, then pitavastatin was added to final concentration of 1,3 and $10 \mu \mathrm{M}$. Twenty four hours after treatment, cells were incubated for Calcein AM/Hoechst Dye solution for 15 min, then fluorescent Calcein retention was measured $20 \mu \mathrm{M}$ Verapamil or cyclosporine A treatments for 20-30 min as positive control of MDR-1 inhibition followed as the 
manufacturer's protocol. The results were expressed as ratio of Calcein AM/ Hoechst signal ( $\mathrm{C} / \mathrm{H}$ ratio). Photomicrographs were taken using fluorescence microscopy.

\section{GBM patients' survival and free disease status relative to MDR-1 (ABCB1) expression}

The GBM patient data were obtained from The Cancer Genome Atlas (TCGA) public data portal, and analyzed using the cBio Cancer Genomics Portal. This system is developed and maintained by the computational biology center of Memorial Sloan-Kettering Cancer Center. We investigated and regrouped GBM patients according their MDR-1 (ABCB1) expression. Firstly, we required the patients/case ID with the MDR-1 expression in all TCGA GBM provisional databases. The mRNA expression $\mathrm{z}$ scores threshold were set as \pm 1 in our analysis. Then, we regrouped all the patients into 3 groups according to their MDR-1 expression as up-regulated, normal, and down-regulated. Finally, we inputted the down-regulated or up-regulated patients ID with normal expressed patients to select patient/case set to analyze patient survival and free disease status data. The Kaplan-Meier curves were drawn based on these analyses.

\section{Animal studies}

The in vivo studies were performed on nude mice to evaluate the drug effects on inhibition of tumor growth. $2 \times 10^{6}$ U87 cells were subcutaneously transplanted into the right and left flanks. Initial tumor growth was monitored every 3 days. Drug administration was initiated when the tumors reached a size of $100-120 \mathrm{~mm}^{3}$. Mice were regrouped into 5 groups of 6 mice each, without significant difference in tumor volume before drug treatment. The mice were treated with either PBS as control, low dose of pitavastatin $(0.5 \mathrm{mg} / \mathrm{kg})$, low dose of irinotecan $(0.5 \mathrm{mg} / \mathrm{kg})$, a combination of pitavastatin $(0.5 \mathrm{mg} / \mathrm{kg})$ and irinotecan $(0.5 \mathrm{mg} / \mathrm{kg})$, or high dose of irinotecan (5 mg/kg). All drugs were injected i.p. in $200 \mu \mathrm{l}$ of PBS, once per day, on a 5-days-on, 2-days-off schedule. Tumors size and mice weight were measured 2 times per week. All mice were sacrificed after tumor sizes reach over $1 \mathrm{~cm}$ in diameter in the control group. Tumor volumes were calculated as (length*width*width/2). After sacrifice, all tumors were disserted and weighted. The animal protocol was approved by UCSD Institutional Animal Care and Use Committee (IACUC).

\section{Statistical analysis}

Activity against GBM cells was assessed by dividing the average number of viable cells (from three replicates per dose) by the average of three controls. At a type I error rate of 0.05 , using a one-sided t-test, we calculated $80 \%$ power to evaluate whether a decrease in mean percent viable cells was significantly lower than $100 \%$, if the observed mean percentage was $91.4 \%$; we conservatively assumed the standard deviation of the percent viable cells was $15 \%$. For significant difference by t-test $(\mathrm{P}<0.05)$, labeled * at the bar graphs.

To quantify the synergism of drug combinations, the drug combination index (CI) was calculated as described by Chou [28]. $\mathrm{ED}_{50}, \mathrm{ED}_{75}$ and $\mathrm{ED}_{90}$ were defined as the drug dose able to inhibit cell growth 50\%, 75\% and 90\%, respectively, for pitavastatin alone, irinotecan alone and mixture of two drugs (various ratios). A CI $<1$ indicates synergy between the two drugs.

\section{Results}

\section{In vitro screening of drugs \\ U87 studies}

The U87 in vitro cell culture platform was used to initially screen the NCC library of 446 small molecules. We calculated percent cell viability as depicted in Figure 1A, and found that 22 drugs reduced viability to less than $50 \%$. Figure $1 \mathrm{~B}$ shows the specific cell viability for each of these 22 compounds. Homoharringtonine and cerivastatin reduced survival to $10 \%$ percent or less, while 9 compounds reduced survival to less than 25\%, 6 drugs reduced survival to less than $35 \%$, and the remainder was associated with a survival of $35-50 \%$. As single agents, all these 22 compounds are more effective in vitro than temozolomide, a widely used drug in GBM treatment (Additional file 1). This result is consistent with previous studies [29,30]. Nine of the 22 compounds producing $<50 \%$ cell survival were more potent than vincristine, a component of a commonly used glioblastoma chemotherapy regimen (procarbazine, lomustine and vincristine - PCV). Similarly, 15 of the 22 compounds were more potent that the commonly used GBM chemotherapeutic irinotecan. As expected, most of the compounds (13 of 22) were antineoplastics and a majority of these (8) oncology drugs are not currently used for the treatment of GBM. Three cardiovascular compounds, cerivastatin, pitavastatin, and nisoldipine showed activity, with the two cholesterollowering agents, cerivastatin and pitavastatin having the greatest effect. The effectiveness of statins prompted us to test a range of commercial available statins; of which, cerivastatin and pitavastatin have the lowest $\mathrm{IC}_{50}$ values (unpublished data).

The two serotonergic pathway inhibitors, sertraline and 5-nonyloxytryptamine also inhibited the survival of U87 cells, which agrees with previously published findings using an adherent GBM stem cell assay [11,12].

\section{A172, LN443 and U118 cells}

To further characterize the most potent compounds identified in our initial screen, we re-screened, using the established cell lines A172, LN443, and U118, the 15 compounds that showed the highest potency with 

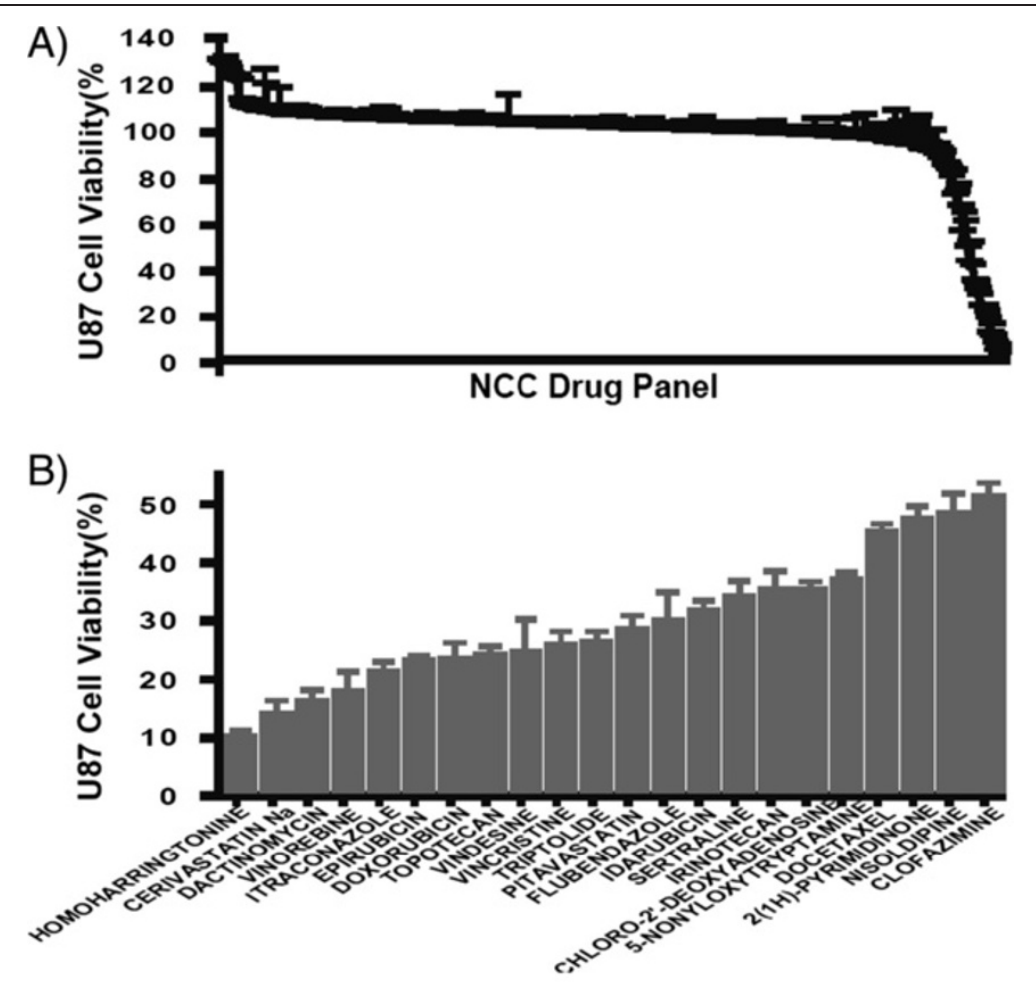

C)

Positive compounds/drugs IC50 of positive compounds and drugs $(\mu \mathrm{M})$

\begin{tabular}{|c|c|c|c|c|}
\hline & $\mathrm{U} 87$ & $\mathrm{~A} 172$ & $\mathrm{LN} 443$ & $\mathrm{U} 118$ \\
\hline Triptolide & 0.006532 & 0.008329 & 0.03076 & 0.01008 \\
\hline Homoharringtonin & 0.022 & $\mathrm{~N} . \mathrm{A}$. & 0.02889 & 0.1419 \\
\hline Dactinmycin & 0.04368 & 0.09932 & 0.01958 & 0.05681 \\
\hline Doxorubicin & 0.05512 & 0.04877 & 0.06286 & 0.04107 \\
\hline Epirubicin & 0.05927 & 0.06217 & 0.04123 & 0.04687 \\
\hline Topotecan & 0.167 & 0.6493 & 0.1358 & 0.184 \\
\hline Itraconazole & 0.389 & 6.974 & $>20 \mathrm{uM}$ & 1.458 \\
\hline Vindesine sulfate & 0.414 & 0.02317 & 0.4948 & 0.67 \\
\hline Cerivastatin & 0.6683 & 0.03166 & 0.02404 & 0.741 \\
\hline Vincrinstine & 1.317 & 0.04202 & 0.8726 & 1.194 \\
\hline Deoxyadenosine & 2.113 & 1.769 & $\mathrm{~N} . \mathrm{A}$. & 0.5897 \\
\hline Pitavastatin & 3.517 & 0.2737 & 0.083 & 1.923 \\
\hline Sertraline & 4.871 & 3.376 & 6.05 & 3.857 \\
\hline Irinotecan & 8.027 & 7.967 & 4.013 & 4.596 \\
\hline Clofazimine & 8.609 & 11.91 & 20.14 & 19.67 \\
\hline Docetaxel & \multicolumn{4}{|c|}{ No dosage effective } \\
\hline
\end{tabular}

Figure 1 NIH clinical collection compounds/drugs screened in vitro with U87 human GBM cells. (A) All 446 compounds in the NCC drug panel ordered according to percent viability after treatment, note the shoulder at the end indicating 22 agents with anti-GBM activity. (B) The 22 positive compounds retested with three replicates. (C) $I_{50}$ of the most active and available compounds screened with four widely used, established GBM cell lines. The predicted BBB value for each compound is shown.

U87 cells (Figure 1C). We found that 8 drugs had greater potency than vincristine in all cell lines tested and 12 drugs had lower $\mathrm{IC}_{50}$ values than irinotecan. We selected 8 FDA approved drugs for further investigation using patient-derived GBM stem cell-like cells (D-Actinomycin, Liposome-encapsulated Doxorubicin/Doxil, Epirubicin, Irinotecan, Pitavastatin, Sertraline, Topotecan and Vincristine).

\section{Stem cell-like GBM lines}

We used GBM stem-like cells derived from surgicallyresected patient samples. Previously, using whole-exome sequencing, we observed global conservation of the patient's tumor genetics in various pre-clinical models, including neurospheres, adherent cells and xenografts [31]. Findings from our study therefore support the 
use of GBM stem-like cells for the development and testing of personalized targeted therapies. In the present study, we used GBM samples from 4 patients that formed neurospheres in culture. Two of these cell lines (SK72 and SK262) also formed adherent cultures. We found that both the neurospheres and adherent cultures expressed equal and high levels of the neural stem cell marker Nestin. Figure 2A shows photomicrographs representative of Nestin staining performed on SK72 neurospheres and SK72 adherent culture. All 8 FDA approved drugs with activity against $\mathrm{U} 87$ cells also had $\mathrm{IC}_{50}$ values lower than two currently used anti-GBM agents, vincrinstine and irinotecan in GBM stem-like cells (neurospheres and adherent cultures). D-actinomycin and epirubicin exhibited the greatest potency (Figure 2B), and the liposomal form of Doxorubicin (Doxil) was less potent than epirubicin even though their $\mathrm{IC}_{50}$ values with U87 cells were virtually the same (data not shown).

The topoisomerase 1B (Topo 1) inhibitor topotecan exhibited potency that significantly surpassed the structurally related Topo 1 inhibitor irinotecan. Similarly, two statins exhibited good activity, which is promising as these drugs have low toxicity and owing to their target pathways may enhance the activity of currently used oncologic agents via synergism. The $\mathrm{IC}_{50}$ for pitavastatin was less than $10 \mu \mathrm{M}$ in most of our cells tested (a range of 1.260 to $55.63 \mu \mathrm{M})$. Similarly, the $\mathrm{IC}_{50}$ of sertraline was in the range of 3.1 to $6.6 \mu \mathrm{M}$.

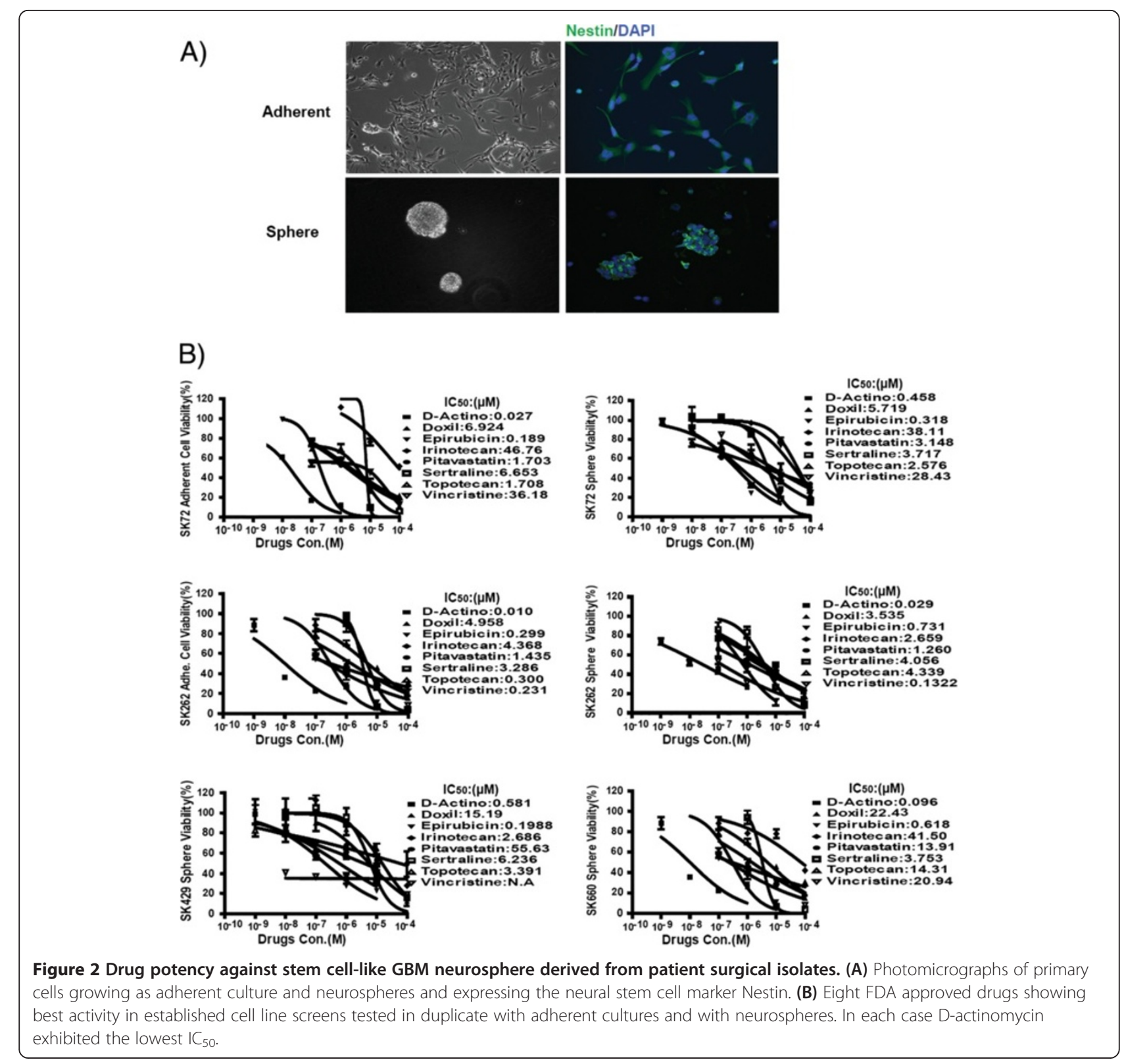




\section{Predicted blood brain barrier permeation values of pitavastatin}

The ability of pitavastatin to cross the $\mathrm{BBB}$ is predicted to be limited as the $-\log \mathrm{BB}$ was calculated as -0.6499 . However, the drug circulates freely in plasma and may enter the enhancing component of tumors via permeation through typically leaky tumor microvessels [25,32].

\section{Effect of pitavastatin on GBM cells}

Considering the effectiveness of statins in our study, specifically pitavastatin in inducing cell death and owing to relatively fewer adverse effects, we decided to explore pitavastatin in detail.

\section{Pitavastatin induces autophagy in GBM cells}

Pitavastatin induced cell morphologic changes, as early as 24 hours, with adherent cells assuming a rounded configuration and detaching from the substrate (Figure 3A). Death of tumor neurospheres was also triggered and these cells arrested in the $G_{0} / G_{1}$ phase after treatment (Figure 3B). $G_{0} / G_{1}$ phase cells were dominant and the proportion of cells in $\mathrm{S}$ phase dramatically decreased.

We found that pitavastatin-treated GBM cells exhibited characteristics consistent with autophagy rather than apoptosis [20,21]. After pitavastatin treatment, GBM cells showed extensive vacuolization, a key feature of cellular macroautophagy $[33,34]$. Further, pitavastatintreated cells stably expressing the GFP-LC3 fusion protein developed a punctate cytoplasmic pattern, suggesting that GFP-LC3 covalently linked to phosphatidylethanolamine (PE) and was inserted into double membrane autophagosomes (Figure 3C).

Morphological observations were confirmed by Westernblot analysis of LC3, which revealed a LC3-I to LC3-II transition, a hallmark of autophagy (Figure 3D) [34,35]. The adherent versus sphere culture conditions did not influence the LC3 transition, which was observed in both U87, U251 adherent stable lines and in the stem cell-like SK72 cell spheres upon pitavastatin treatment. Furthermore, increasing concentrations of pitavastatin enhanced LC3-I to II transition (Figure 3D, right panel). In addition, Annexin staining failed to detect apoptosis after pitavastatin (data not shown) treatment. Caspase-3/7 activity was not detectable via fluorescence or by Westernblot analysis (Figure 3E). We could not entirely exclude the possibility that pitavastatin induced cell apoptosis by caspase independent pathways; however the cell cycle analysis shown in Figure $3 \mathrm{~B}$ argued against this hypothesis, as it did not reveal a sub-G1 population, characteristic of apoptotic cells. The mechanism(s) of cell death induced by pitavastatin still needs more detailed investigation. Further, whether other statins can also trigger autophagy in human GBM cells remains to be determined, and this may depend, in part, on whether adherent cells or neurosphere cultures are assayed.

To elucidate the possible mechanisms by which pitavastatin decreases cell survival, we also used a virtual tumor cell technology. This is an in silico analysis using a comprehensive and dynamic representation of signaling and metabolic pathways underlying tumor physiology (based on CellWorks Inc. technology, Additional file 2). Using this platform, we tested the effect of pitavastatin on two GBM cell lines (A172 and U251) using genomic profiles. In silico modeling data predicted a significantly increase in autophagy makers in both GBM cells following pitavastatin treatment (Additional file 3).

\section{Drug combinations}

We then tested 12 drugs along with pitavastatin to investigate possible additive or synergistic effects. In these combinations tested using U87 cells, only irinotecan and pitavastatin displayed a synergistic effect, with effective lowering of $\mathrm{IC}_{50}$ for both compounds (Figure 4A). This synergistic effect was further confirmed in U118 and SK72 cells, using a concentration range of pitavastatin, which showed a dramatic 40-70 fold lowering of the $\mathrm{IC}_{50}$ compared to irinotecan alone (Figure 4B). Drug combination index $(\mathrm{CI})$, calculated at $\mathrm{ED}_{50}, \mathrm{ED}_{75}$ and $\mathrm{ED}_{90}$, ranged from $0.28-0.76$ for U118 cells $0.55-0.87$ for U87 cells and 0.41-1.29 for SK72 cells demonstrating a moderate-tostrong synergism between irinotecan and pitavastatin at various drug concentrations in all three GBM cell lines. Importantly, the addition of pitavastatin reversed the resistance of the primary SK72 neurosphere cells to irinotecan, causing a decrease in its $\mathrm{IC}_{50}$ from $30 \mu \mathrm{M}$ to $1.5 \mu \mathrm{M}$.

\section{Enhancement of irinotecan via suppression of MDR-1 by pitavastatin}

Irinotecan induces apoptosis, which is primarily responsible for its anti-tumor activity [36]. Although pitavastatin as a single agent did not induce apoptosis, in combination with irinotecan, it enhanced U87 caspase-3 activity as compared to irinotecan alone, both at 12 and 24 hours (Figure 4C). The major mechanism of drug resistance in GBM is the over-expression of the multi-drug resistance protein (MDR-1; p-glycoprotein 1 or $A B C B 1)$, seen in the $\mathrm{BBB}$ and neuroepithelial tumors such as GBM [37]. Multiple studies have established that MDR-1 is responsible for decreased drug accumulation in multidrug-resistant GBM cells [38,39]. Interestingly, pitavastatin is a substrate of MDR-1 [40]. We observed that MDR-1 gene transcription levels correlated directly with irinotecan concentration (Figure 4D). However, after combined pitavastatin and irinotecan treatment, the $140 \mathrm{KD}$ MDR-1 band increased in intensity, suggesting MDR- glycosylation is suppressed, which attenuates the production of functional MDR-1 (Figure 4E) [41]. 

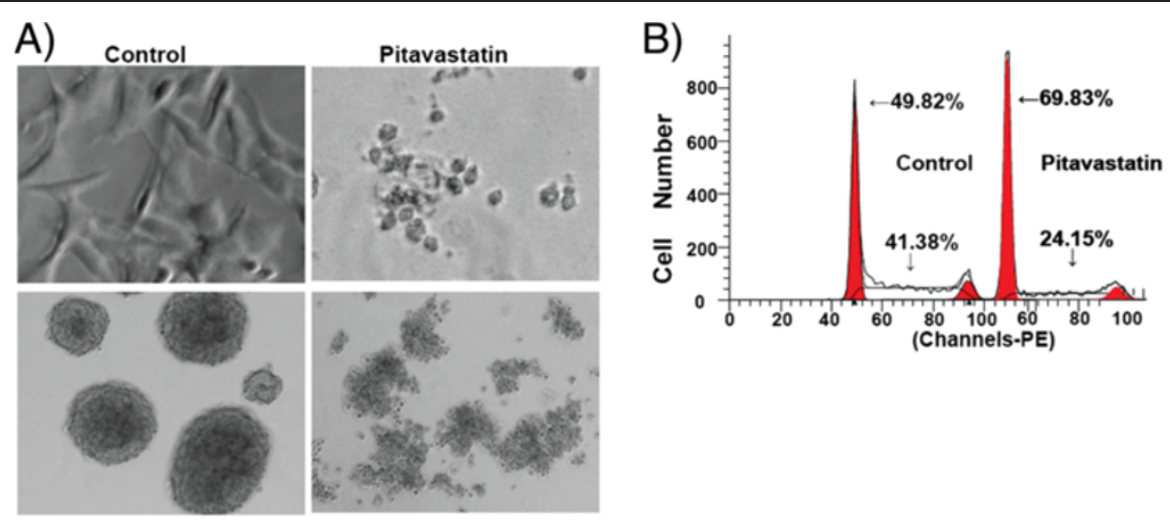

C)

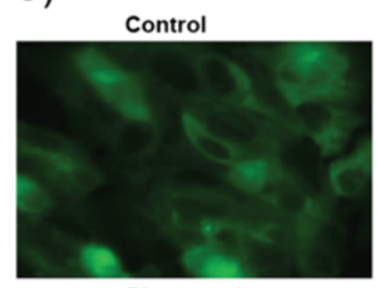

Pitavastatin

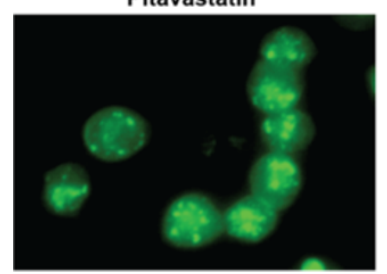

D)

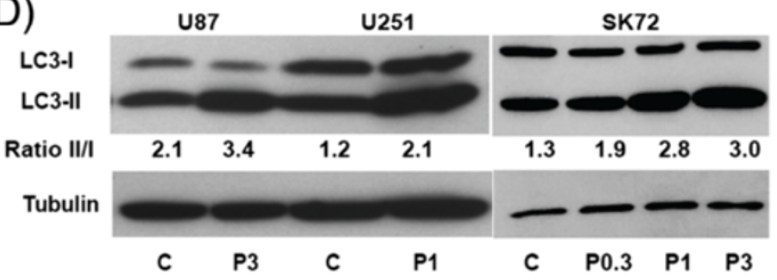

E)

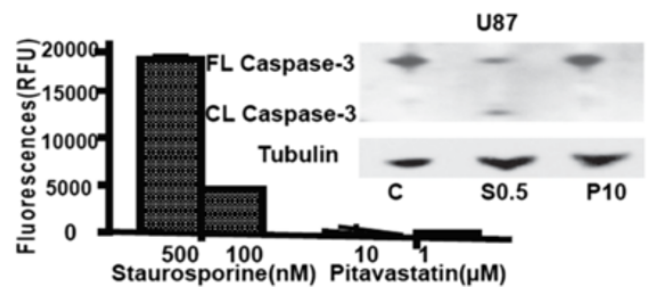

Figure 3 Pitavastatin induces autophagy associated cell death for GBM. (A) Pitavastatin induced SK72 glioma primary cell death in adherent and neurosphere cultures. Note rounding of cells at 24 h. (B) FACS analysis showing that pitavastatin treated U87 cells were arrested in $\mathrm{G}_{0} / \mathrm{G}_{1}$ phase with a much lower percentage of the total population in S phase. (C) In GFP-LC3 transduced-U87 GBM cells, pitavastatin induced a punctuate GFP-LC3 distribution, which is a characteristic of autophagy. (D) Western blot showing that pitavastatin induced the transformation of LC3 protein from isoform I to II in U87, U251 and SK72 cells. (Pitavastatin concentrations in $\mu \mathrm{M}$ ). LC3I/II was calculated with NIH ImageJ software (E) Caspase-3/7 activity in U87 cells induced by staurosporine for 8 hours detected by the Apo-One fluorescence kit. In contrast, no caspase $3 / 7$ activity was seen with pitavastatin treatment during 2 days. Inset: Western blot illustrating that there was no detectable cleavage $(\mathrm{CL})$ of caspase-3 after pitavastatin treatment $(\mathrm{P}, 10 \mu \mathrm{M})$; Staurosporine treatment $(\mathrm{S}, 0.5 \mu \mathrm{M})$ was used as a positive control and was associated with caspase-3 cleavage (CL).

\section{Pitavastatin inhibited MDR-1 function}

As shown in Figure 4D and E, pitavastatin induced MDR1 mRNA and decreased glycosylation of MDR-1 protein. To elucidate the effect of pitavastatin on MDR-1 function, we evaluated the drug exclusion capability directly, using the Calcein-AM assay. As showed in Figure 4F, after statin treatment, both U87 and SK72 GBM cells showed increased intracellular amounts of the MDR-1 substrate (Calcein AM), indicating that pitavastatin may inhibit drug-exclusion mediated by MDR-1. The MDR-1 inhibition was directly proportional to pitavastatin concentration. This result suggests that the increased caspase activity, observed in cells treated with irinotecan in combination with pitavastatin, may be due to its MDR-1 inhibitory effects, which in turn caused accumulation of irinotecan (Figure 4C).
Down-regulation of MDR-1 (ABCB1 gene) expression correlates with overall survival and longer disease-free status

In TCGA dataset, of the 243 GBM samples profiled, 43 showed down-regulation of MDR-1/ABCB1 (Figure 5A), 15 were amplified for MDR-1/ABCB1 and 34 had MDR$1 / A B C B 1$ up-regulation. This result suggested that the MDR-1 transcription levels are variable and may be regulated by the tumor microenvironment. In all 173 cases with normal MDR-1 expression level (blue-line), the median survival was 14.1 months $(n=149)$ whereas in patients with MDR-1 down-regulation (red-line), it was increased to 23.2 months $(\mathrm{P}=0.027$; Figure $5 \mathrm{~B})$. Further, progression-free survival increased from 6.67 months in patients with normal MDR-1 to 11.54 months in case of 
A)

Drugs IC 50 with and without Pitavastatin $(2 \mu \mathrm{M})$
\begin{tabular}{|c|c|c|}
\hline & & $+2 \mu$ M Pita \\
\hline Triptolide & 0.008631 & 0.03376 \\
\hline Homoharringtonin & 0.01627 & 0.03507 \\
\hline Dactinmycin & 0.01108 & 0.03525 \\
\hline Doxorubicin & 0.07342 & 0.08975 \\
\hline Epirubicin & 0.05927 & 0.1214 \\
\hline Topotecan & 0.4721 & 0.5862 \\
\hline Itraconazole & 0.3865 & 3.794 \\
\hline Vindesine & 0.8284 & 1.04 \\
\hline Cerivastatin & 0.6683 & N.D. \\
\hline Vincrinstine & 0.9156 & 1.442 \\
\hline Deoxyadenosine & 2.113 & N.D. \\
\hline Sertraline & 4.871 & 7.309 \\
\hline Pitavastatin & 5.76 & \\
\hline Irinotecan & 8.025 & 3.356 \\
\hline Clofazimine & 8.609 & N.D. \\
\hline
\end{tabular}

C)

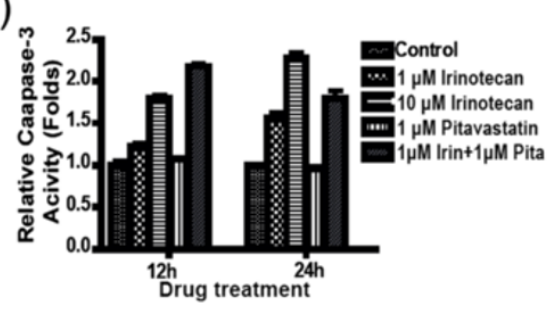

D)

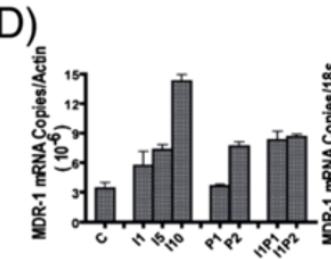

B)

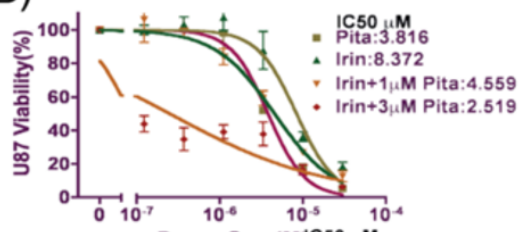

$-100-2$ Drugs Con.(M/C50 $\mu$ M M
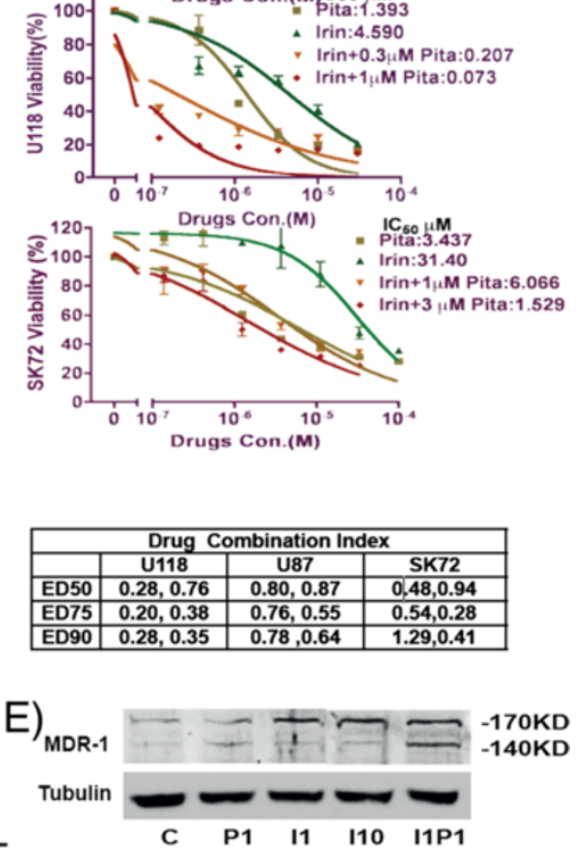
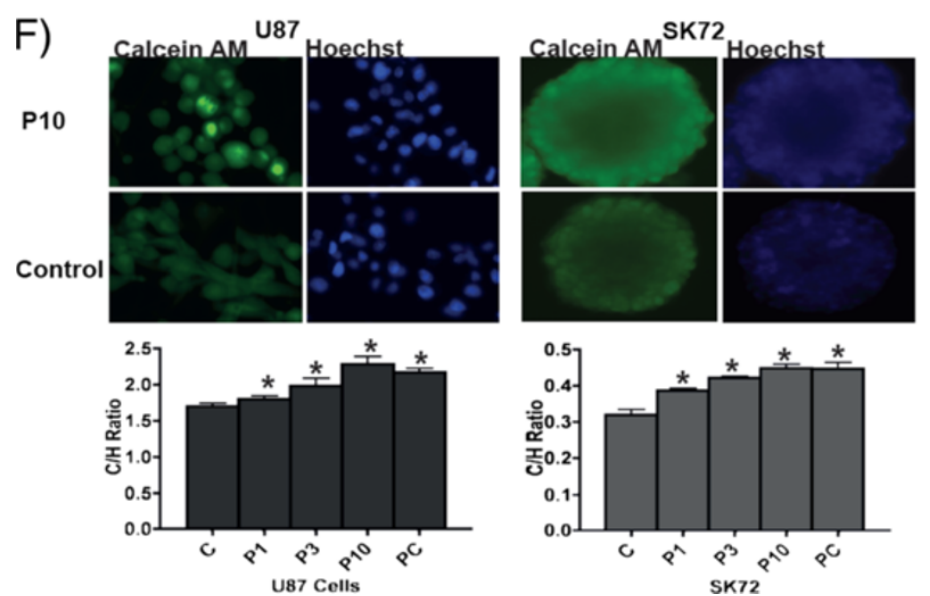

Figure 4 Pitavastatin and irinotecan exhibit synergism in terms of inducing GBM cell death in vitro. (A) U87 IC 50 values for various compounds with and without pitavastatin $(2 \mu \mathrm{M})$, note that irinotecan $I_{50}$ was reduced by $50 \%$. (B) Pitavastatin, sharply lowered the $I C_{50}$ of irinotecan in U118, U87 cells and SK72. The combination index calculated at ED $\mathrm{E}_{50}, \mathrm{ED}_{75}$ and $\mathrm{ED}_{90}$. Data were calculated at two pitavastatin concentrations. (C) Escalation of apoptosis by addition of pitavastatin to irinotecan, particularly after 12 hours of incubation. (D) Pitavastatin and irinotecan applied singly induce MDR-1 mRNA transcription as shown by qRT-PCR, and when applied together the level of transcription is only slightly greater than with irinotecan alone. (E) Western blot analysis indicating that after irinotecan treatment, U87 expressed full length MDR-1 (170 KD), but in combination with pitavastatin, the band density of unglycosylated MDR-1 (140 KD) increased dramatically. Unglycosylated MDR-1 cannot be transported inside the cell and is essentially non-functional. (F) After pitavastatin treatment, U87 cell and SK72 cells were accumulated more Calcein AM and showed higher fluorescent signal than control by inhibited the MDR-1 function. 

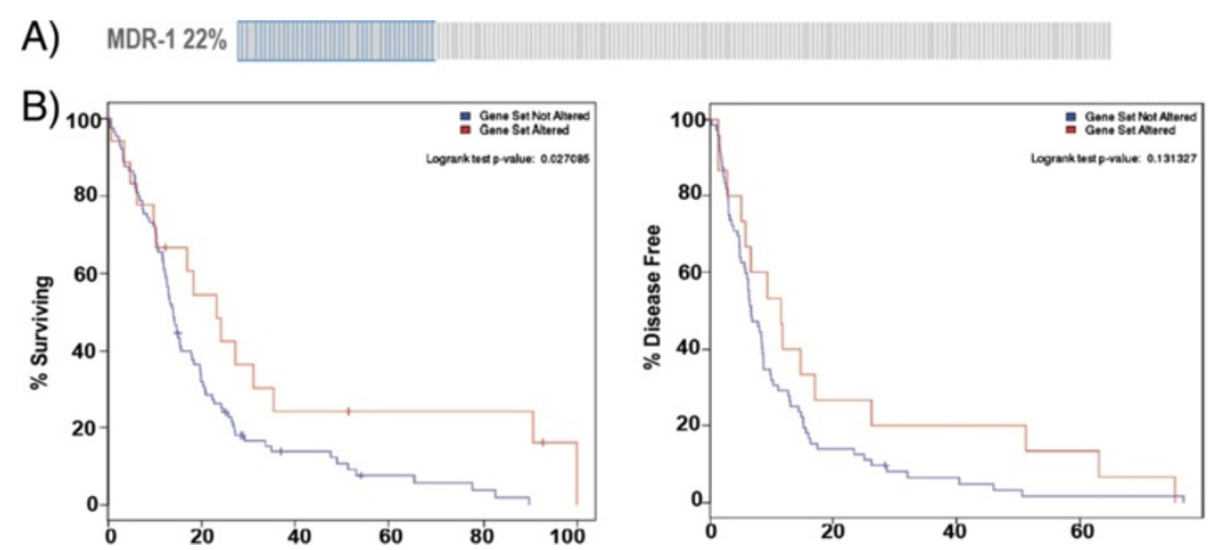

Figure 5 Down-regulation of MDR-1 (gene ABCB1) predicted better survive for GBM patients. A) Oncoprint mRNA expression map generated using cBio Cancer Genomics. 22\% of a total of 243 GBM patients showed down-regulated MDR-1 (ABCB1) expression B) Down-regulation of MDR-1 (red-line) increases overall patient survival as the median survival (blue-line) increased from 14.1 months to 23.2 months in Kaplan-Meier curves $(P<0.05)$ and patients' median progression-free survival increased from 6.67 to 11.54 months $(P=0.13)$.

MDR-1 down-regulation. For patients with MDR-1 upregulation or gene amplification, there was no difference in overall or progression-free survival when compared to controls (data not shown). These data strongly suggest that MDR-1 inhibition following treatment with statins may have a beneficial effect in GBM patients.

\section{Combination of Pitavastatin and Irinotecan enhances anti-tumor efficacy in vivo}

To evaluate the in vivo anti-cancer effect of pitavastatin and irinotecan, we treated xenograft mouse models implanted with U87 cells with either single agent or combination. As shown in Figure 6A, low dose pitavastatin or irinotecan did not affect tumor growth. In contrast, $0.5 \mathrm{mg} / \mathrm{kg}$ pitavastatin in combination with $0.5 \mathrm{mg} / \mathrm{kg}$ irinotecan significantly attenuated tumor growth $(\mathrm{P}<0.01)$ compared to both the control group and the low-dose single drug treatment groups (one week /5 drug treatment). Tumor measurements after sacrificing the mice at day 32 confirmed that combination treatment significantly reduced tumor size and weight $(\mathrm{P}<0.001$; Figure $6 \mathrm{~A}$, right panel). Interestingly, irinotecan administered as a single agent but at a dose 10-times higher than that used in the combination treatment group was also very potent in inhibiting in vivo U87 tumor growth. However, such high doses were associated with significant drug toxicity, as indicated by severe weight loss in drug-treated mice (Figure 6B). In contrast, the body weights of mice receiving a combination of pitavastatin and low-dose irinotecan increased 3-4 gram steadily similar to that seen in control and the low dose drug treatment groups during the whole study duration. Moreover, tumor cell proliferation decreased dramatically as showed by the Ki67 staining in Figure 6C.

\section{Discussion}

In the present study, we sought to screen a library of FDA-approved compounds to rapidly identify new, nonGBM drugs that could be readily introduced into GBM clinical trials. Using a platform that employed a wide range of human GBM lines, including clinically relevant patientderived primary GBM lines, our screening uncovered 22 compounds from different classes with anti-neoplastic activity in GBM. Among others, the cardiovascular drugs statins showed high efficacy in reducing tumor growth both in vitro and in vivo, drawing our attention to these relatively non-toxic cholesterol lowering drugs. The present study demonstrates the potency of pitavastatin relative to other statins. Importantly, our results demonstrated that co-administration of pitavastatin with low-dose chemotherapy, greatly increased the potency of the latter, lowering the $\mathrm{IC}_{50}$ values for irinotecan by 40 - to 70 -fold, with few adverse effects. Experimentally, we found that statins independently induced autophagy in GBM and that statins may potentiate chemotherapeutic agents by inhibiting MDR-1 function. This was consistent with in silico screening results using our virtual tumor cell technology, which suggested that pitavastatin affects cell viability by inducing autophagy.

Cholesterol has a key role in cell membranes, cell metabolism, cell signaling and has been implicated in tumor development and progression. Therefore, as cholesterollowering agents, questions about the anti-tumor effects of statins have been already posed [42,43]. Statins decrease cholesterol levels by inhibiting the enzyme HMG-CoA reductase in the liver. In addition, mevalonate, and isoprenoid intermediates such as geranylgeranylpyrophosphate (GGPP) and farnesylpyrophosphate (FPP) in the cholesterol synthesis pathway are also depleted after statin treatment 

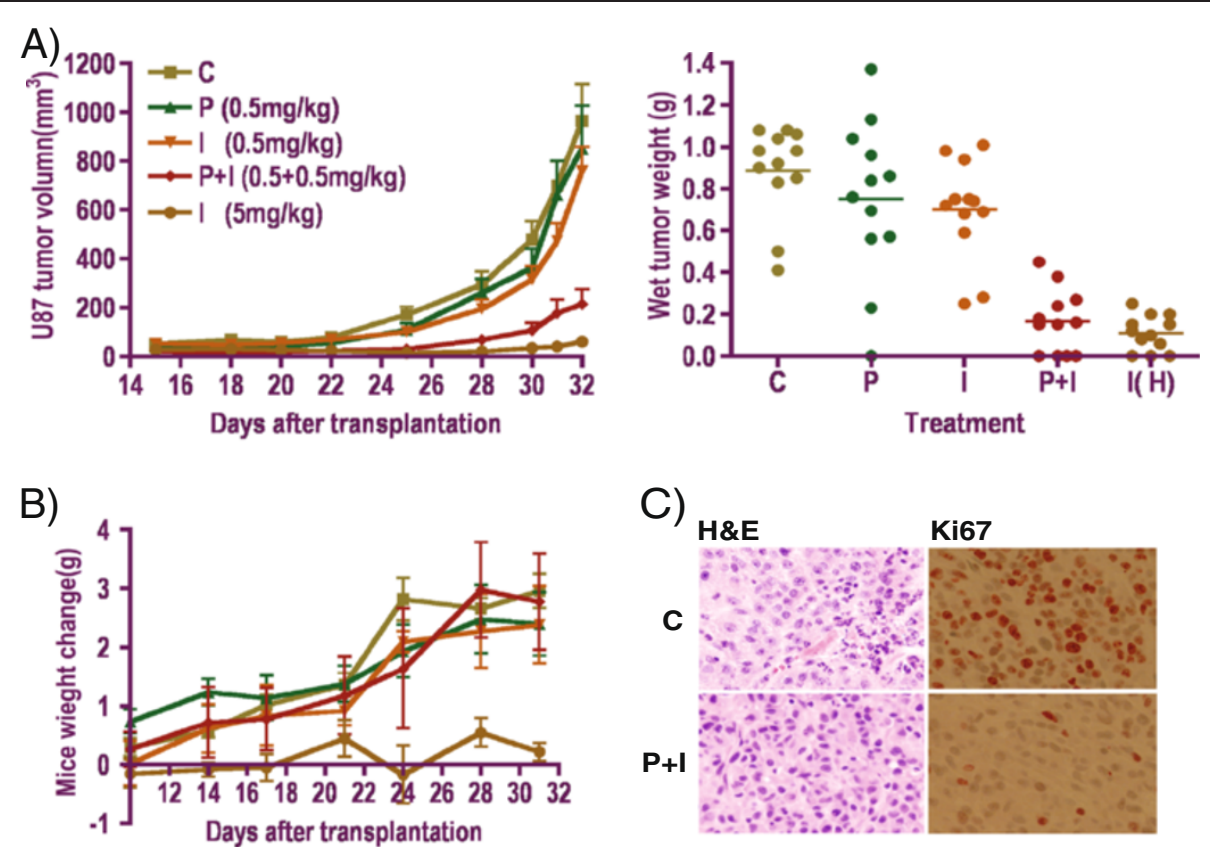

Figure 6 Co-administration of low dose of pitavastatin and irinotecan inhibited tumor growth in vivo. A) U87 tumor growth was inhibited by combination treatment but not by low dose of single drug; tumor volume and weight dramatically decreased following combination treatment when compared to control and single drug-treated groups. B) A combination of low dose pitavastatin and irinotecan showed no toxicity whereas high dose irinotecan induced mice body weight loss. C) H\&E and Ki-67 staining of control and combination treatment of in vivo U87 tumor model, showed a much lower proliferation in the tumor cells after combination treatment.

[44]. Another intermediate, dolichol, an essential substrate for protein $\mathrm{N}$-glycosylation, is also blocked by statins $[45,46]$. Considering that GBMs are highly proliferative taking up large quantities of cholesterol, potentially they may be vulnerable to statin treatment $[47,48]$. However, the mechanism of sensitivity of GBM to statins has not been elucidated. Recent studies have shown that statins may have an anti-GBM effect in xenograft mouse models, by targeting the low-density lipoprotein receptor (LDLR), inducing apoptosis via ERK/AKT pathway $[20,47]$. Other data hypothesize that statins may inhibit tumor growth by inducing autophagy via the NF- $\mathrm{kB}$ pathway in human colon cancer cell line [49]. Our data obtained in both stable cell lines and primary patient samples clearly demonstrated that pitavastatin induced macro-autophagy in GBM cells $[20,21]$. Further experiments are now ongoing to investigate the signaling pathway(s) involved in this effect.

Importantly, we have shown that pitavastatin potentiated the anti-tumor effects of low-dose irinotecan, a topoisomerase inhibitor. Pitavastatin is know to be a substrate of the multi-drug resistance protein, MDR-1, which is overexpressed in GBM upon drug treatment and is partly responsible for the resistance of GBM to chemotherapy. Our data indicate that, in combination with irinotecan, pitavastatin suppressed glycosylation of MDR-1, thereby inhibiting its function and allowing irinotecan to accumu- late intracellularly $[37,39,40]$. Accumulation of irinotecan is likely responsible for the increased apoptosis in the presence of pitavastatin. The MDR-1 expression in cancer cells can be a significant obstacle to the success of chemotherapy. Many MDR-1 inhibitors have been extensively tested in clinical trials but the results have been inconclusive. According to TCGA data, down-regulated ABCB1 (MDR-1) predicted better survival of GBM patients. Combining a statin with a chemotherapeutic agent represents a powerful, potential strategy for circumventing resistance and significantly enhancing efficacy. Here we have confirmed that pitavastatin may improve the therapeutic response to TOPO-1 inhibitors, by inhibiting MDR-1 function, and may be beneficial for GBM patients. It remains to be determined whether other statins exert a similar or a different anti-neoplastic mechanism as compared to pitavastatin, and whether different subtypes of GBM have different sensitivity to pitavastatin or display other mechanisms for statin actions. GBM is a complex and heterogeneous disease that likely accounts for the different results obtained across various studies.

Irinotecan is broadly used in solid cancer therapy, especially in combination with other drugs $[50,51]$. In clinical use, the toxicity of irinotecan is generally manageable and reversible [52,53]. However, in some patients it may lead to severe side effects, such as diarrhea and neutropenia that can be life threatening. In our animal model, 
co-administration of pitavastatin allowed for a reduced dosage of irinotecan and avoided drug toxicity at higher dosage. These data indicate a new strategy to develop better irinotecan-based drug combination.

Based on the promising results of our present study, we are now undertaking additional preclinical studies of GBM to optimize dosing and characterize efficacy, thus providing a solid basis for a clinical trial with pitavastatin and irinotecan for the treatment of glioblastoma patients.

\section{Additional files}

Additional file 1: Figure S1. U87 cell viability after NCC drug treatment. U87 cell has various drug responses after single dose of all 446 NCC drug treatments. We showed all data in here which summarized in Figure $1 \mathrm{~A}$. The Top 22 hits were showed in Figure 1B.

\section{Additional file 2: In silico modeling protocol.}

Additional file 3: Figure S2. In Silico modeling predicts pitavastatininduced autophagy in GBM cell lines. We simulated the effect of pitavastatin on the virtual tumor cell that modeled GBM cell lines A172 and U251. Our simulation demonstrated increased expression of autophagy and related pathway markers in both these cell lines.

\section{Competing interests}

The authors declare that they have no competing interests.

\section{Authors' contributions}

PFJ and SK designed the study and submitted paper. PFJ performed all experiments and collected all data. TA and SV performed in silico analysis. VF, SP, XC help analysis in vitro data. MG collected patients' samples. RM, IBS, YC, NN helped on collected in vivo data. MM wrote the first draft of paper. SCP and SK revised the manuscript. All authors read and approved the final manuscript.

\section{Acknowledgements}

The authors wish to thank Gabi Gomez for technical assistance in the laboratory.

\section{Financial support}

Grants from NIH (NIH 3P30CA023100-25S8), National Brain Tumor Society David Cook Chair of Research and from Barbara and Joseph Ajello trust fund, Tuttleman Family Foundation and Boston Fire Department/Kenney Foundation to S. Kesari.

\section{Author details}

${ }^{1}$ Translational Neuro-Oncology Laboratories, Moores Cancer Center, UC San Diego, La Jolla, CA 92093, USA. ²Department of Neurosciences, UC San Diego, La Jolla, CA 92093, USA. ${ }^{3}$ Moores Cancer Center, UC San Diego, 3855 Health Sciences Drive, La Jolla, CA 92093, USA. ${ }^{4}$ CellWorks Inc., Irvine, CA, USA.

Received: 25 December 2013 Accepted: 10 January 2014 Published: 17 January 2014

\section{References}

1. Wen PY, Kesari S: Malignant gliomas in adults. N Engl J Med 2008, 359:492-507.

2. Duffner PK: Risk factors for cognitive decline in children treated for brain tumors. Eur J Paediatr Neurol 2010, 14:106-115.

3. Salacz ME, Watson KR, Schomas DA: Glioblastoma: Part I. Current state of affairs. Mo Med 2011, 108:187-194.

4. Fueyo J, Gomez-Manzano C, Yung WK: Advances in translational research in neuro-oncology. Arch Neurol 2011, 68:303-308.

5. Sharma A, Jacob A, Tandon M, Kumar D: Orphan drug: development trends and strategies. J Pharm Bioallied Sci 2010, 2:290-299.

6. Omuro AM, Faivre S, Raymond E: Lessons learned in the development of targeted therapy for malignant gliomas. Mol Cancer Ther 2007, 6:1909-1919.
7. Jeon SH, Kim SH, Kim Y, Kim YS, Lim Y, Lee YH, Shin SY: The tricyclic antidepressant imipramine induces autophagic cell death in U-87MG glioma cells. Biochem Biophys Res Commun 2011, 413:311-317.

8. Kast RE: Glioblastoma: synergy of growth promotion between CCL5 and NK-1R can be thwarted by blocking CCL5 with miraviroc, an FDA approved anti-HIV drug and blocking NK-1R with aprepitant, an FDA approved anti-nausea drug. J Clin Pharm Ther 2010, 35:657-663.

9. Galli R, Binda E, Orfanelli U, Cipelletti B, Gritti A, De Vitis S, Fiocco R, Foroni C, Dimeco F, Vescovi A: Isolation and characterization of tumorigenic, stem-like neural precursors from human glioblastoma. Cancer Res 2004, 64:7011-7021.

10. Singh SK, Hawkins C, Clarke ID, Squire JA, Bayani J, Hide T, Henkelman RM, Cusimano MD, Dirks PB: Identification of human brain tumour initiating cells. Nature 2004, 432:396-401.

11. Pollard SM, Yoshikawa K, Clarke ID, Danovi D, Stricker S, Russell R, Bayani J, Head R, Lee M, Bernstein M, et al: Glioma stem cell lines expanded in adherent culture have tumor-specific phenotypes and are suitable for chemical and genetic screens. Cell Stem Cell 2009, 4:568-580.

12. Diamandis P, Wildenhain J, Clarke ID, Sacher AG, Graham J, Bellows DS, Ling EK, Ward RJ, Jamieson LG, Tyers $M$, et al: Chemical genetics reveals a complex functional ground state of neural stem cells. Nat Chem Biol 2007, 3:268-273.

13. Lee J, Kotliarova S, Kotliarov Y, Li A, Su Q, Donin NM, Pastorino S, Purow BW, Christopher N, Zhang $W$, et al: Tumor stem cells derived from glioblastomas cultured in bFGF and EGF more closely mirror the phenotype and genotype of primary tumors than do serum-cultured cell lines. Cancer Cell 2006, 9:391-403.

14. Reynolds BA, Rietze RL: Neural stem cells and neurospheres-re-evaluating the relationship. Nat Methods 2005, 2:333-336.

15. Shen G, Shen F, Shi Z, Liu W, Hu W, Zheng X, Wen L, Yang X: Identification of cancer stem-like cells in the C 6 glioma cell line and the limitation of current identification methods. In Vitro Cell Dev Biol Anim 2008, 44:280-289.

16. Chen J, McKay RM, Parada LF: Malignant glioma: lessons from genomics, mouse models, and stem cells. Cell 2012, 149:36-47.

17. Vik-Mo EO, Sandberg C, Joel M, Stangeland B, Watanabe Y, Mackay-Sim A, Moe MC, Murrell W, Langmoen IA: A comparative study of the structural organization of spheres derived from the adult human subventricular zone and glioblastoma biopsies. Exp Cell Res 2011, 317:1049-1059.

18. Reardon DA, Friedman HS, Powell JB Jr, Gilbert M, Yung WK: Irinotecan: promising activity in the treatment of malignant glioma. Oncology (Williston Park) 2003, 17:9-14.

19. Prados MD, Lamborn K, Yung WK, Jaeckle K, Robins HI, Mehta M, Fine HA, Wen PY, Cloughesy T, Chang S, et al: A phase 2 trial of irinotecan (CPT-11) in patients with recurrent malignant glioma: a North American Brain Tumor Consortium study. Neuro Oncol 2006, 8:189-193.

20. Yanae M, Tsubaki M, Satou T, Itoh T, Imano M, Yamazoe Y, Nishida S: Statin-induced apoptosis via the suppression of ERK1/2 and Akt activation by inhibition of the geranylgeranyl-pyrophosphate biosynthesis in glioblastoma. J Exp Clin Cancer Res 2011, 30:74.

21. Hindler K, Cleeland CS, Rivera E, Collard CD: The role of statins in cancer therapy. Oncologist 2006, 11:306-315.

22. Brown JM, Giaccia AJ: The unique physiology of solid tumors: opportunities (and problems) for cancer therapy. Cancer Res 1998, 58:1408-1416

23. Juillerat-Jeanneret $L$ : The targeted delivery of cancer drugs across the blood-brain barrier: chemical modifications of drugs or drug-nanoparticles? Drug Discov Today 2008, 13:1099-1106.

24. Yu SP, Yang XJ, Zhang B, Ming HL, Chen C, Ren BC, Liu ZF, Liu B: Enhanced invasion in vitro and the distribution patterns in vivo of CD133+ glioma stem cells. Chin Med J (Engl) 2011, 124:2599-2604.

25. Claes A, Wesseling P, Jeuken J, Maass C, Heerschap A, Leenders WP: Antiangiogenic compounds interfere with chemotherapy of brain tumors due to vessel normalization. Mol Cancer Ther 2008, 7:71-78.

26. Nicolazzo JA, Charman SA, Charman WN: Methods to assess drug permeability across the blood-brain barrier. J Pharm Pharmacol 2006 58:281-293

27. Fan Y, Unwalla R, Denny RA, Di L, Kerns EH, Diller DJ, Humblet C: Insights for predicting blood-brain barrier penetration of CNS targeted molecules using QSPR approaches. J Chem Inf Model 2010, 50:1123-1133.

28. Chou TC: Theoretical basis, experimental design, and computerized simulation of synergism and antagonism in drug combination studies. Pharmacol Rev 2006, 58(3):621-681. 
29. Mathieu V, De Neve N, Le Mercier M, Dewelle J, Gaussin JF, Dehoux M, Kiss R, Lefranc F: Combining bevacizumab with temozolomide increases the antitumor efficacy of temozolomide in a human glioblastoma orthotopic xenograft model. Neoplasia 2008, 10:1383-1392.

30. Mrugala MM, Chamberlain MC: Mechanisms of disease: temozolomide and glioblastoma-look to the future. Nat Clin Pract Oncol 2008, 5:476-486.

31. Yost SE, Pastorino S, Rozenzhak S, Smith EN, Chao YS, Jiang P, Kesari S, Frazer KA, Harismendy O: High-resolution mutational profiling suggests the genetic validity of glioblastoma patient-derived pre-clinical models. PLoS One 2013, 8:e56185.

32. Fukumura D, Jain RK: Tumor microenvironment abnormalities: causes, consequences, and strategies to normalize. J Cell Biochem 2007, 101:937-949.

33. Gonzalez-Polo RA, Boya P, Pauleau AL, Jalil A, Larochette N, Souquere $S$, Eskelinen EL, Pierron G, Saftig P, Kroemer G: The apoptosis/autophagy paradox: autophagic vacuolization before apoptotic death. J Cell Sci 2005, 118:3091-3102.

34. Boya P, Gonzalez-Polo RA, Casares N, Perfettini JL, Dessen P, Larochette N, Metivier D, Meley D, Souquere S, Yoshimori T, et al: Inhibition of macroautophagy triggers apoptosis. Mol Cell Biol 2005, 25:1025-1040.

35. Shi R, Weng J, Zhao L, Li XM, Gao TM, Kong J: Excessive autophagy contributes to neuron death in cerebral ischemia. CNS Neurosci Ther 2012, 18:250-260.

36. Whitacre CM, Zborowska E, Willson JK, Berger NA: Detection of poly(ADP-ribose) polymerase cleavage in response to treatment with topoisomerase I inhibitors: a potential surrogate end point to assess treatment effectiveness. Clin Cancer Res 1999, 5:665-672.

37. Nabors MW, Griffin CA, Zehnbauer BA, Hruban RH, Phillips PC, Grossman SA, Brem H, Colvin OM: Multidrug resistance gene (MDR1) expression in human brain tumors. J Neurosurg 1991, 75:941-946.

38. Takeba Y, Sekine S, Kumai T, et al: Irinotecan-induced apoptosis is inhibited by increased P-glycoprotein expression and decreased p53 in human hepatocellular carcinoma cells. Biol Pharm Bull 2007, 30:1400-1406.

39. Nakai E, Park K, Yawata T, Chihara T, Kumazawa A, Nakabayashi H, Shimizu K: Enhanced MDR1 expression and chemoresistance of cancer stem cells derived from glioblastoma. Cancer Invest 2009, 27:901-908.

40. Shirasaka Y, Suzuki K, Shichiri M, Nakanishi T, Tamai I: Intestinal absorption of HMG-CoA reductase inhibitor pitavastatin mediated by organic anion transporting polypeptide and P-glycoprotein/multidrug resistance 1. Drug Metab Pharmacokinet 2011, 26:171-179.

41. Schinkel AH, Kemp S, Dolle M, Rudenko G, Wagenaar E: N-glycosylation and deletion mutants of the human MDR1 P-glycoprotein. J Biol Chem 1993, 268:7474-7481.

42. Nielsen SF, Nordestgaard BG, Bojesen SE: Statin use and reduced cancer- related mortality. N Engl J Med 2012, 367:1792-1802.

43. Thurnher M, Nussbaumer O, Gruenbacher G: Novel aspects of mevalonate pathway inhibitors as antitumor agents. Clin Cancer Res 2012, 18:3524-3531.

44. Goldstein JL, Brown MS: Regulation of the mevalonate pathway. Nature 1990, 343:425-430.

45. McCarty MF: Suppression of dolichol synthesis with isoprenoids and statins may potentiate the cancer-retardant efficacy of IGF-I down-regulation. Med Hypotheses 2001, 56:12-16.

46. Williams AB, Li L, Nguyen B, Brown P, Levis M, Small D: Fluvastatin inhibits FLT3 glycosylation in human and murine cells and prolongs survival of mice with FLT3/ITD leukemia. Blood 2012, 120:3069-3079.

47. Guo D, Reinitz F, Youssef M, Hong C, Nathanson D, Akhavan D, Kuga D, Amzajerdi AN, Soto $H$, Zhu S, et al: An LXR agonist promotes GBM cell death through inhibition of an EGFR/AKT/SREBP-1/LDLR-dependent pathway. Cancer Discov 2011, 1:442-456.

48. Shibata Y, Matsumura A, Yoshida F, Yamamoto T, Nakai K, Nose T, Sakata I, Nakajima S: Competitive uptake of porphyrin and LDL via the LDL receptor in glioma cell lines: flow cytometric analysis. Cancer Lett 2001, 166:79-87.

49. Tsuboi $Y$, Kurimoto $M$, Nagai $S$, et al: Induction of autophagic cell death and radiosensitization by the pharmacological inhibition of nuclear factor-kappa B activation in human glioma cell lines. J Neurosurg 2009, 110:594-604.

50. Weekes J, Lam AK-Y, Sebesan S, Ho Y-H: Irinotecan therapy and molecular targets in colorectal cancer: a systemic review. World J Gastroenterol 2009, 15:3597-3602
51. Vredenburgh JJ, Desjardins A, Reardon DA, Friedman HS: Experience with irinotecan for the treatment of malignant glioma. Neuro Oncol 2009, 11:80-91.

52. Hahn KK, Wolff JJ, Kolesar JM: Pharmacogenetics and irinotecan therapy. Am J Health Syst Pharm 2006, 63:2211-2217.

53. de Jong FA, de Jonge MJ, Verweij J, Mathijssen RH: Role of pharmacogenetics in irinotecan therapy. Cancer Lett 2006, 234:90-106.

doi:10.1186/1479-5876-12-13

Cite this article as: Jiang et al:: Novel anti-glioblastoma agents and therapeutic combinations identified from a collection of FDA approved drugs. Journal of Translational Medicine 2014 12:13.

\section{Submit your next manuscript to BioMed Central and take full advantage of:}

- Convenient online submission

- Thorough peer review

- No space constraints or color figure charges

- Immediate publication on acceptance

- Inclusion in PubMed, CAS, Scopus and Google Scholar

- Research which is freely available for redistribution

Submit your manuscript at www.biomedcentral.com/submit
C) Biomed Central 